\title{
Cartilha de direitos de pessoas idosas e/ou com deficiência: uma abordagem baseada em Design inclusivo e infografia
}

Booklet about rights of elderly and people with disabilities: an approach based on inclusive design and infography

\author{
Camila Medina, Caroline Spósito, Eduardo Jannone da Silva, Natália Barreto Frederigue \\ Lopes, Adriane Lima Mortari Moret, Regina Tangerino de Souza Jacob
}

design da informação, design inclusivo, infografia, deficiência

\begin{abstract}
Mudanças na legislação brasileira relacionadas aos Direitos das Pessoas com Deficiência, asseguram a este grupo de indivíduos uma gama de direitos fundamentais da mais alta valia, que geram maior respeito aos Direitos Humanos. Entretanto, o baixo nível de letramento funcional em saúde desta população e o formalismo da linguagem técnico-jurídica do sistema normativo brasileiro dificultam o acesso e compreensão de tais informações/direitos pelos destinatários. Assim, o objetivo deste estudo foi elaborar uma Cartilha Informativa contendo os principais direitos colocados à disposição das pessoas com deficiência e/ou idosas, bem como as providências a serem adotadas pelos interessados em acessá-los. Para tal, legislações especificas, elencadas por tema, foram selecionadas e estruturadas de forma simplificada e direta, mediante princípios do design inclusivo e recursos da infografia. A cartilha será utilizada pelos Assistentes Sociais para informar e orientar pacientes e acompanhantes usuários dos serviços da Divisão de Saúde Auditiva do Hospital de Reabilitação de Anomalias Craniofaciais e da Clínica de Fonoaudiologia da Faculdade de Odontologia de Bauru, ambas vinculadas à Universidade de São Paulo. A concepção da referida Cartilha Informativa representa efetivo instrumento colocado à disposição das Pessoas com Deficiência, na busca de se promover uma sociedade cada vez mais livre, justa, solidária e inclusiva.
\end{abstract}

Information design. Inclusive design, infography, disability

Changes in Brazilian legislation related to the Rights of Persons with Disabilities assure this group of individuals a range of fundamental rights of highest value, which generate greater respect for Human Rights. However, the low level of functional literacy in health of this population and the formalism of legal-technical language of Brazilian normative system make it difficult for recipients to access and understand such information / rights. Thus, the purpose of this study was to prepare an Information Booklet containing the main rights made available to disabled and elderly people, as well as measures to be adopted by those interested in accessing them. For this, specific legislations, listed by topic, were selected and structured in a simplified and direct way, through principles of inclusive design and infographic resources. The booklet will be used by the Social Assistants to inform and guide patients and their accompanying who uses of the services of the Auditory Health Division of the Hospital for Rehabilitation of Craniofacial Anomalies and the Speech Therapy Clinic of Bauru School of Dentistry, both linked to University of São Paulo. Information Booklet's design represents an effective tool available to people with disabilities, in search to promote a society increasingly free, supportive and inclusive.

\section{Introdução}

Segundo a Pesquisa Nacional de Saúde - PNS de 2013, cerca de 6,2\% da população brasileira possuía ao menos um tipo de deficiência. Neste levantamento, foram considerados quatro tipos de deficiências - a visual, que atinge 3,6\% dos brasileiros; a física, com 1,3\%; a auditiva, com $1,1 \%$ e a intelectual, que acomete $0,8 \%$ da população. Em todas elas, observa-se maior prevalência na parcela da população com 60 anos ou mais e/ou os indivíduos sem instrução ou com fundamental incompleto.

Anais do 8 CIDI e 8 CONGIC

Guilherme Santa Rosa; Cristina Portugal (orgs.)

Sociedade Brasileira de Design da Informação - SBDI

Natal | Brasil | 2017

ISBN 978-85-212-1305-5
Proceedings of the $8^{\text {th }} \mathrm{CIDI}$ and $8^{\text {th }}$ CONGIC

Guilherme Santa Rosa; Cristina Portugal (orgs.)

Sociedade Brasileira de Design da Informação - SBDI

Natal| Brazil | 2017

ISBN 978-85-212-1305-5 
No Brasil, segundo o Instituto Brasileiro de Geografia e Estatística -IBGE (IBGE, 2015), em 2010, havia mais de 20,5 milhões de pessoas com mais de 60 anos no país - estima-se que esse número mais que triplique nos próximos 20 anos. O crescimento do índice de envelhecimento da população traz consigo importantes implicações e desafios para a sociedade, incluindo a área da saúde.

Mudanças na legislação brasileira como a incorporação da Convenção Internacional sobre os Direitos das Pessoas com Deficiência da ONU por meio da emenda constitucional no Artigo $5^{\circ}$, § $3^{\circ}$ em $2009^{1}$ da Constituição brasileira e a Lei Brasileira de Inclusão da Pessoa com Deficiência (Lei Federal $\left.n^{\circ} 13.146 / 2015\right)^{2}$, asseguram, a tal grupo de indivíduos uma gama de direitos fundamentais da mais alta valia e que gera maior respeito aos Direitos Humanos.

Contudo, o sistema normativo brasileiro faz o uso do formalismo através de linguagem técnico-jurídica, o que dificulta o acesso e compreensão pelos destinatários de tais informações/direitos- razão pela qual a utilização de ferramentas como as Cartilhas Informativas tem sido cada vez mais recorrente.

De acordo com a Organização Mundial da Saúde (OMS), as "competências cognitivas e sociais que determinam a motivação e a capacidade dos indivíduos para obter acesso, compreender e utilizar a informação em meios que promovem e mantém uma boa saúde" (WHO, 1998, p. 10) são caracterizadas pelo Letramento Funcional em Saúde (LFS). A habilidade do indivíduo em compreender conceitos em saúde e aplicá-los em seu cotidiano é de extrema importância para o sucesso do tratamento e seu bem-estar. No Brasil, Passamai (2013) verificou que os níveis de compreensão textual e numérica em saúde foram extremamente baixos, o que pode comprometer a saúde individual e coletiva. O LFS limitado dos indivíduos impacta negativamente na habilidade para ler e compreender orientações fornecidas sobre cuidados com sua saúde ou necessitarão de ajuda para compreendê-las.

Ações referentes ao Design Inclusivo devem atender à variabilidade da funcionalidade física, sensorial e cognitiva das pessoas idosas e/ou com deficiência. Ele deve ser centrado no utilizador, abranger a diversidade e contribuir para a melhora da qualidade de vida. Projetar com inclusividade significa incluir aqueles que prossivelmente seriam ignoradas no processo de design (Pinheiro \& Silva, 2010). Considera-se, portanto, o Design Inclusivo, uma prática projetual "de respeito aos direitos humanos e de defesa de condições de igualdade de oportunidades" (Simões \& Bispo, 2006 p. 8) que busca a concepção de produtos inclusivos.

A infografia é uma estratégia de comunicação que utiliza ferramentas do Design Gráfico e da Informação para estruturar o conteúdo, tornando-o mais acessível de forma mais fácil e eficaz. Trata-se de "...uma macro-linguagem, que surge da combinação entre mensagem verbal e imaginética" (Fassina, Cavalcante \& Andrade, 2009, p. 287).

Assim sendo, busca-se com a concepção de uma Cartilha Informativa, voltada às pessoas idosas e/ou com deficiência do município de Bauru, baseada em conceitos do Design da Informação, Design Inclusivo e infografia na busca de se promover uma sociedade cada vez mais livre, justa, solidária e inclusiva.

\section{Parâmetros para desenvolvimento de produtos inclusivos na área de saúde}

Para elaborar a cartilha, foi realizado levantamento bibliográfico acerca de recomendações e suas respectivas justificativas para o desenvolvimento de materiais instrucionais inclusivos para a área de saúde, divididos por categoria (adaptado de Medina, 2017), aqui separado de acordo com a organização dos elementos textuais e gráficos, conforme tabela 1 e 2.

\footnotetext{
${ }^{1}$ Brasil, Poder Executivo. Decreto n6.949/2009 , de 25 de agosto de 2009, Brasília, DF. < http://www.planalto.gov.br/ccivil_03/_ato2007-2010/2009/decreto/d6949.htm>

${ }^{2}$ Brasil, Poder Legislativo. Lei Federal no 13.146/2015, de 6 de julho de 2015, Brasília, DF. < http://www.planalto.gov.br/ccivil_03/_ato2015-2018/2015/lei/l13146.htm>
} 
Tabela 1: Recomendações para desenvolvimento de materiais instrucionais na área da saúde (elementos textuais)

\begin{tabular}{l|l|l} 
Categoria & Recomendação & Justificativa \\
\hline Conteúdo & $\begin{array}{l}\text { Restringir a quantidade de informações } \\
\text { Limitar objetivos de aprendizagem evitando } \\
\text { informações que possam confundir o leitor. }\end{array}$ & $\begin{array}{l}\text { É necessário minimizar a quantidade de } \\
\text { informações para não sobrecarregar a } \\
\text { memória de trabalho. }\end{array}$ \\
\hline Linguagem & $\begin{array}{l}\text { Evitar uso excessivo de palavras incomuns e } \\
\text { termos técnicos. } \\
\text { Usar palavras e sentenças simples. }\end{array}$ & $\begin{array}{l}\text { Indivíduos com diferentes graus de } \\
\text { letramento tem menor dificuldade em } \\
\text { compreender materiais com linguagem } \\
\text { simples. }\end{array}$ \\
\hline $\begin{array}{l}\text { Organiza- } \\
\text { ção }\end{array}$ & $\begin{array}{l}\text { Informações consideradas mais importantes } \\
\text { devem ser apresentadas primeiro. } \\
\text { Criar hierarquia entre títulos e subtítulos para } \\
\text { organizar os conteúdos. }\end{array}$ & $\begin{array}{l}\text { Os leitores desejam encontrar } \\
\text { informações com mais facilidade e } \\
\text { rapidez e devem distinguir facilmente os } \\
\text { títulos do restante do texto. }\end{array}$ \\
\hline $\begin{array}{l}\text { Estímulo à } \\
\text { leitura e } \\
\text { motivação }\end{array}$ & $\begin{array}{l}\text { Usar linguagem e cotidiano do público alvo. } \\
\text { Usar imagens e exemplos culturalmente } \\
\text { apropriados }\end{array}$ & $\begin{array}{l}\text { Deve haver uma identificação entre o } \\
\text { usuário e o material, bem como deve-se } \\
\text { evitar elementos que causem qualquer } \\
\text { incompatibilidade na assimilação do } \\
\text { mesmo. } \\
\text { O material deve promover vontade de ler } \\
\text { e fornecer respostas rápidas aos leitores. }\end{array}$ \\
\hline
\end{tabular}

Fonte: Bull et al.2001; Caposecco; Hickson \& Meyer, 2011 e 2014; Hoffman \& Worral, 2004; MHRA, 2014 
Tabela 2: Recomendações para desenvolvimento de materiais instrucionais na área da saúde (elementos gráficos)

\begin{tabular}{|c|c|c|}
\hline Categoria & Recomendação & Justificativa \\
\hline \multirow[t]{5}{*}{ Tipografia } & $\begin{array}{l}\text { Usar fontes com } 12 \text { a } 14 \text { pontos. } \\
\text { Evitar itálico e sublinhado. }\end{array}$ & $\begin{array}{l}\text { Pessoas idosas possuem déficit de visão e/ou } \\
\text { cognição que podem afetar a capacidade de } \\
\text { leitura. }\end{array}$ \\
\hline & $\begin{array}{l}\text { Não usar fontes complicadas, } \\
\text { decorativas e cursivas. }\end{array}$ & Fontes mais detalhadas dificultam a leitura. \\
\hline & $\begin{array}{l}\text { Usar fontes padrão, com preferência } \\
\text { pelas fontes sem serifa. }\end{array}$ & $\begin{array}{l}\text { Fontes padrão são mais facilmente } \\
\text { reconhecidas. }\end{array}$ \\
\hline & Usar caixa alta e baixa. & $\begin{array}{l}\text { Uso de caixa alta e baixa é mais confortável e } \\
\text { eficiente }\end{array}$ \\
\hline & $\begin{array}{l}\text { Espacejamento entre as linhas adequado } \\
\text { (idêntico ao tamanho da fonte) }\end{array}$ & Espacejamentos pequenos dificultam a leitura. \\
\hline \multirow[t]{6}{*}{ Layout } & Usar papel fosco. & O reflexo da luz no papel dificulta a leitura. \\
\hline & $\begin{array}{l}\text { Diagramar impresso em orientação } \\
\text { paisagem }\end{array}$ & $\begin{array}{l}\text { A orientação paisagem maximiza o espaço } \\
\text { disponível. }\end{array}$ \\
\hline & $\begin{array}{l}\text { Usar um formato que permita a } \\
\text { distribuição do conteúdo de maneira } \\
\text { confortável, mantendo-se textos e figuras } \\
\text { em tamanho legível. } \\
\text { A peça também precisa ser fácil de } \\
\text { localizar, transportar e ler }\end{array}$ & $\begin{array}{l}\text { Impressos pequenos podem exigir tipografias } \\
\text { em corpos pouco legíveis e ilustrações } \\
\text { reduzidas, dificultando a visualização e leitura. }\end{array}$ \\
\hline & $\begin{array}{l}\text { Usar fontes escuras sobre suporte claro } \\
\text { (o inverso pode ser usado para realçar } \\
\text { informações importantes). }\end{array}$ & $\begin{array}{l}\text { Mais legíveis: Fundo branco, cinza claro ou } \\
\text { amarelo no objeto azul escuro ou preto; ou } \\
\text { fundo cinza escuro, azul escuro, vermelho ou } \\
\text { preto, e o objeto branco ou amarelo. }\end{array}$ \\
\hline & Usar alinhamento à esquerda & $\begin{array}{l}\text { Blocos justificados podem causar problemas } \\
\text { no espaço entre palavras quando as colunas } \\
\text { de texto são mais estreitas. }\end{array}$ \\
\hline & Uso de espaços em branco nos textos & $\begin{array}{l}\text { Espaços em branco criam sensação de } \\
\text { clareza sobre a informação a ser apresentada }\end{array}$ \\
\hline \multirow[t]{2}{*}{ llustração } & Usar ilustrações a traço. & $\begin{array}{l}\text { Estas ilustrações possuem menos elementos } \\
\text { que possam distrair o leitor. }\end{array}$ \\
\hline & $\begin{array}{l}\text { Usar boxes, flechas e etiquetas e } \\
\text { pictogramas. }\end{array}$ & $\begin{array}{l}\text { Tais elementos gráficos, usados de maneira } \\
\text { correta, chamam a atenção para importantes } \\
\text { partes ou ações. }\end{array}$ \\
\hline
\end{tabular}

Fonte: Alessandrini, 1984; Caposecco; Hickson \& Meyer, 2011 e 2014; Hoffman \& Worral, 2004; Silva, 2012; MHRA, 2014

Diante disso, foi definido que a cartilha deveria nortear-se por estes princípios para que seu objetivo informativo possa ser mais facilmente alcançado. 


\section{Cartilha Informativa - Concepção}

O levantamento das leis e a estruturação destas em módulos é fruto de outro estudo em andamento, voltado às pessoas com deficiência e/ou idosos, especialmente àqueles que frequentam serviços públicos de alta complexidade da cidade de Bauru (Jacob et al, 2017).

Participou da execução da cartilha, uma equipe de profissionais das áreas do direito, psicologia, serviço social, fonoaudiologia e design. O público alvo deste projeto consiste em pacientes e acompanhantes usuários dos serviços da Divisão de Saúde Auditiva do Hospital de Reabilitação de Anomalias Craniofaciais e da Clínica de Fonoaudiologia da Faculdade de Odontologia de Bauru, ambas vinculadas à Universidade de São Paulo. A expectativa é que sejam informados, através da presente cartilha, uma média aproximada de 40 pacientes/acompanhantes por dia, durante os atendimentos prestados pelos profissionais do Serviço Social.

Foram abordados 9 tópicos acerca das leis que beneficiam pessoas com deficiência e/ou idosos. A cartilha contou com 12 páginas, sendo uma para cada lei, a capa e informações sobre a equipe de desenvolvimento e instituições de apoio.

Após a estruturação do conteúdo, foi definida a identidade visual do projeto, especialmente norteada pela criação de pictogramas e infográficos e uso de cores e tipografia de fácil compreensão. $\mathrm{Na}$ capa, a opção do uso de qrcode para a áudio-descrição foi a estratégia utilizada para contemplar e incluir os deficientes visuais. $O$ formato final fechado do impresso foi o tamanho A5, preferido em um grupo focal realizados com pacientes adultos e idosos deficientes auditivos da cidade de Bauru (Medina, 2007).

As figuras abaixo ilustram a versão final da cartilha.

Figura 1. Capa da cartilha

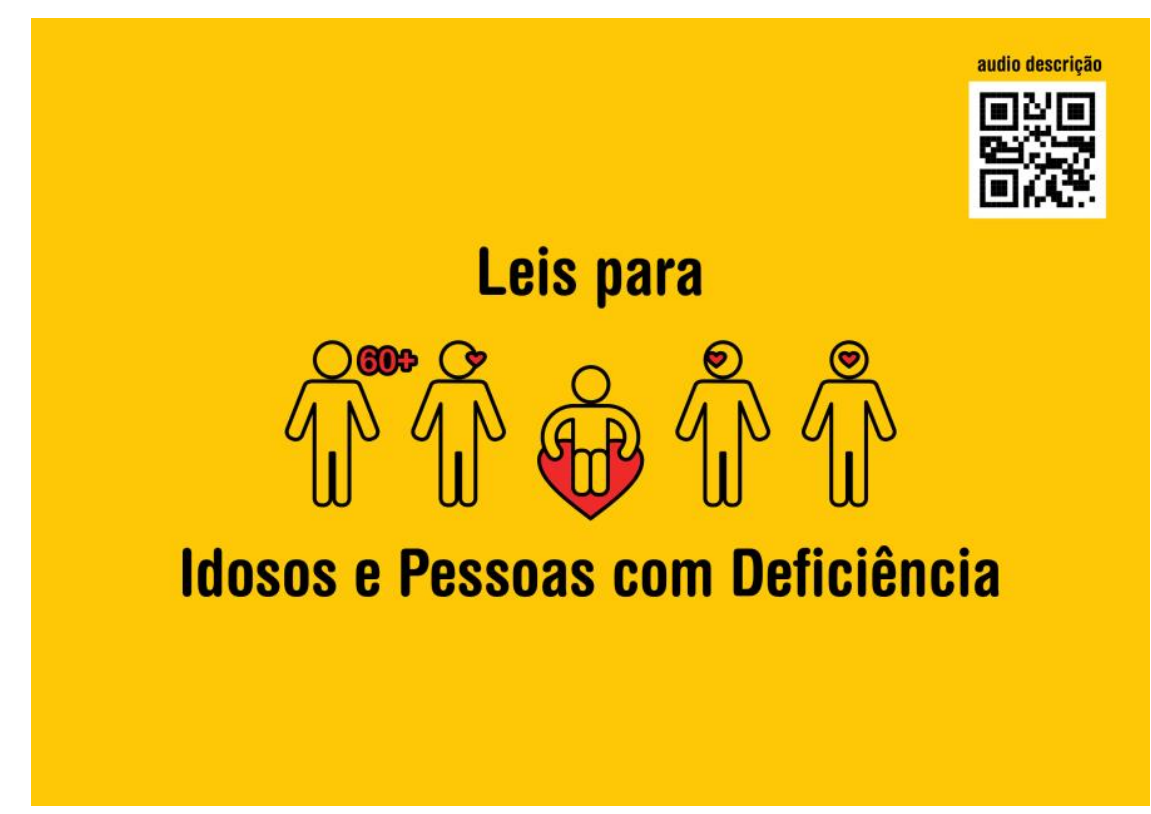


Figura 2. Página "Passe Livre Interestadual"

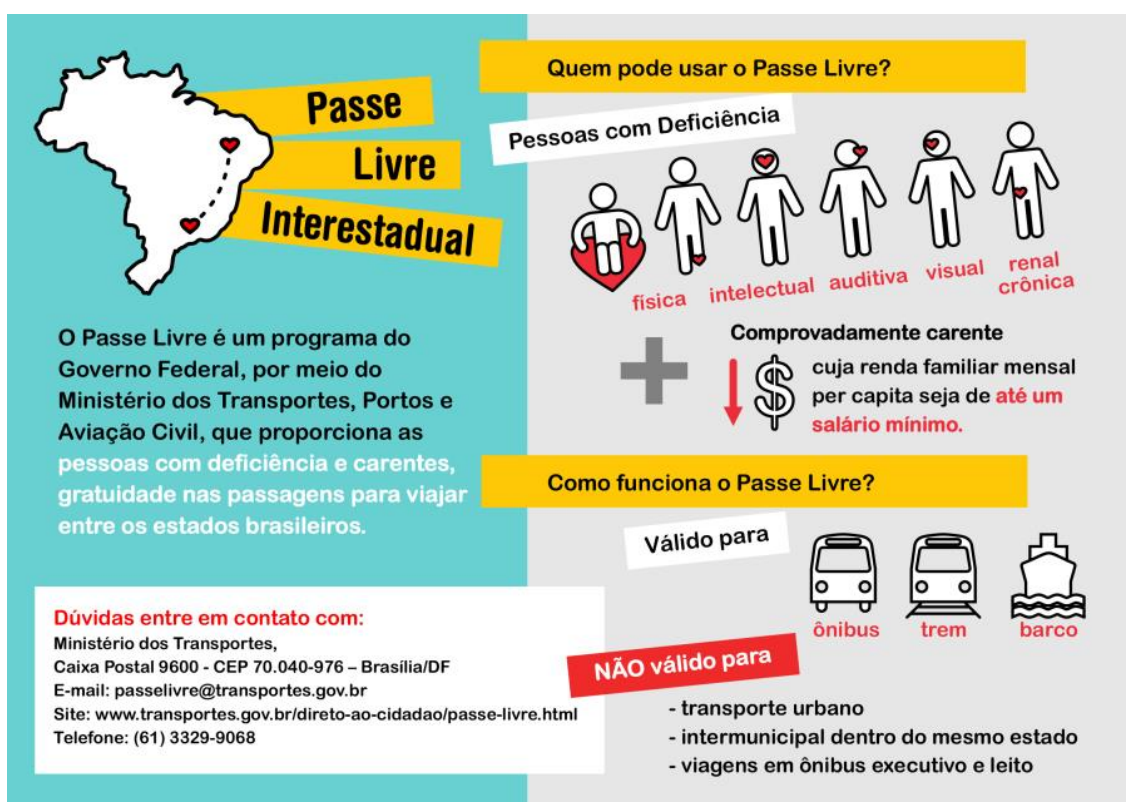

Figura 3. Página "Aposentadoria para pessoa om deficiência"

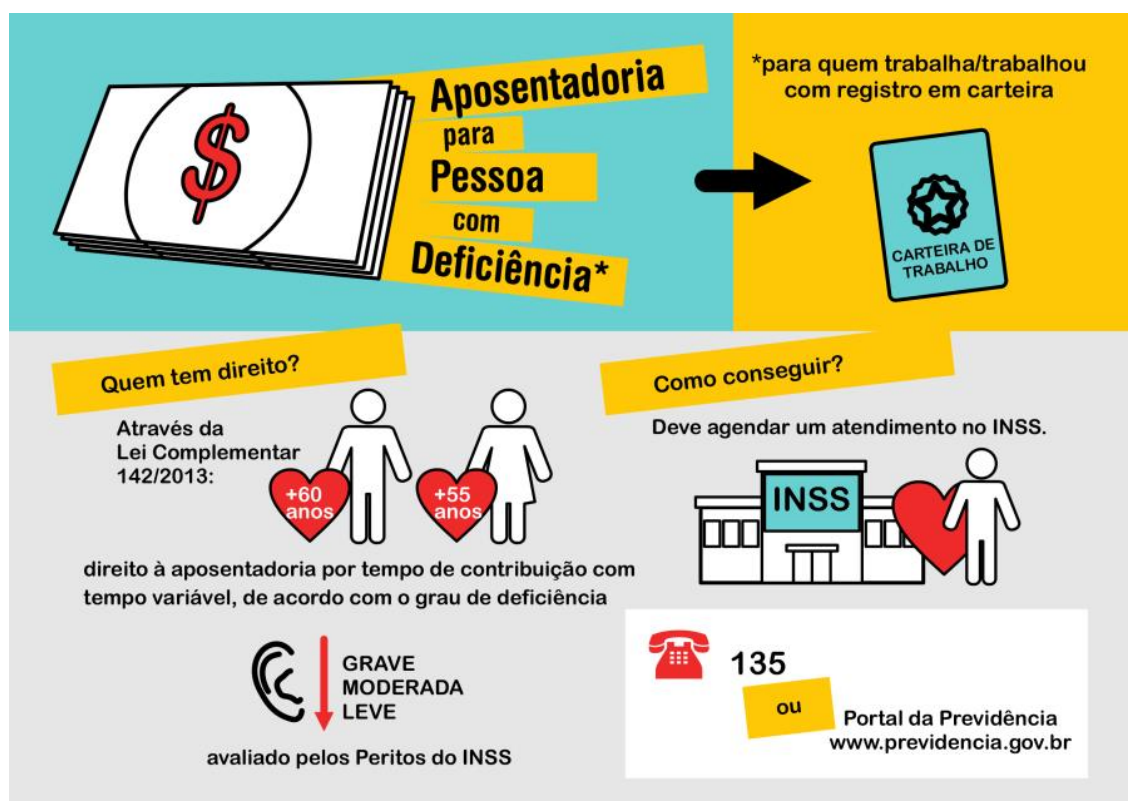

\section{Discussão e considerações finais}

A presente cartilha busca informar às pessoas com deficiência e/ou idosos seus direitos e obrigações, bem como a maneira de acessá-los, promovendo cidadania e tornando-os multiplicadores de tais informações.

Para tal, a restrição do uso de termos técnicos e artifícios da infografia foram estratégias usadas para tornar o impresso inclusivo a quem ele é destinado. Em alguns países, iniciativas de agências governamentais auxiliam no desenvolvimento de materiais instrucionais na área da saúde, indicando estratégias para a compreensão de tais informativos, como um guia de boas práticas para a criação de instruções ao paciente sobre o uso de medicamentos no Reino Unido (MHRA, 2014). Um guia de boas práticas, fornecido pelo governo brasileiro, que indique 
a correta forma de transmissão da informação na área da saúde para a população, poderia facilitar na concepção de tais materiais instrucionais.

No tocante da identidade visual deste projeto, em relação as cores, houve a preocupação do contraste entre elas e entre os elementos gráficos e textuais. Silva (2012) estudou o impacto da alteração da visão de pessoas idosas em produtos impressos e concluiu que, para tornar a percepção das cores mais fácil, o contraste entre objeto e fundo deve ser considerado. $O$ correto uso das cores e o uso de tipografia em tamanho maior também auxiliam aqueles com baixa visão e com deficiências congênitas. Outro aspecto a se destacar, foi a criação de um padrão de pictogramas relativos às deficiências, cujo propósito foi o de afastar a estigmatização. A inclusão social destas pessoas é ainda muito incipiente em nosso país e ações como o acatamento à legislação vigente; uso da mídia, da cibercultura e de novas tecnologias são passos fundamentais para mudar este quadro (Maciel, 2000).

Este estudo possibilitou identificar as necessidades, tanto em relação a transmissão de informações sobre as leis de grande valia às pessoas com deficiência e/ou idosos, quanto à forma como ela é transmitida.

\section{Referências}

FASSINA, U., Cavalcante, A.L.B. \& Andrade, R.C. 2009. Reflexões sobre a complementariedade imagem e texto, e o seu papel na criação da linguagem da infografia. In: Anais do $2^{\circ}$ Encontro Nacional dos Estudos da Imagem; Londrina, Brasil <http://www.uel.br/eventos/eneimagem/anais/caderno_de_resumos_site.pdf>

IBGE, 2013 - Instituto Brasileiro de Geografia e Estatística. Pesquisa Nacional de Saúde - PNS: percepção do estado de saúde, estilos de vida e doenças crônicas - Brasil, Grandes Regiões e Unidades de Federação Rio de Janeiro: Instituto Brasileiro de Geografia e Estatística. $<\mathrm{ftp}: / /$ ftp.ibge.gov.br/PNS/2013/pns2013.pdf>

IBGE, 2015 - Instituto Brasileiro de Geografia e Estatística. Síntese de Indicadores Sociais Uma análise das condições de vida da população brasileira. Rio de Janeiro: Instituto Brasileiro de Geografia e Estatística.

<http://biblioteca.ibge.gov.br/visualizacao/livros/liv95011.pdf>

JACOB, R.T.S. et al. 2017. Cartilha de Direitos das Pessoas com Deficiência (Projeto de Pesquisa: categoria direitos fundamentais). In. $2^{\circ}$ EDITAL SANTANDER/USP/FUSP de Direitos Fundamentais e Políticas Públicas / Pró-Reitoria de Cultura e Extensão Universitária.

MACIEL, M.R.C. 2000. Portadores de deficiência: a questão da inclusão social. São Paulo em Perspectiva, v.14, n.2: 51-56.

MEDINA C. 2017. Interface entre Design e Fonoaudiologia: material instrucional impresso voltado aos usuários de aparelho de amplificação sonora individual. Bauru. Dissertação de mestrado não publicada. Faculdade de Odontologia de Bauru USP.

MHRA, 2014. Medicines and Healthcare products Regulatory Agency - Gov.UK. Best practice guidance on patient information leaflets.

<https://www.gov.uk/government/uploads/system/uploads/attachment_data/file/328405/Best_ practice_guidance_on_patient_information_leaflets.pdf>

PASSAMAI, M.P.B.; Sampaio, H.A.C. \& Lima, J.W. 2013. Letramento Funcional em Saúde de Adultos no Contexto do Sistema Único de Saúde. Fortaleza: EdUECE.

SILVA, J.C.P.; Paschoarelli, L.C. \& SILVA, F.M. Design ergonônimo: estudos e aplicações. Bauru: FAAC - Universidade Estadual Paulista.

SIMÕES, J.F. \& Bispo, R. 2012. Design Inclusivo: acessibilidade e usabilidade em produtos, serviços e ambientes. Lisboa: CTP Produção.

WHO, 1998 - World Health Organiztion. Health promotion glossary. Geneva: WHO. 


\section{Sobre o(a/s) autor(a/es)}

Camila Medina, mestre, FOB-USP, Brasil <camila@fob.usp.br>

Caroline Spósito, mestranda, HRAC-USP, Brasil <csposito@usp.br>

Eduardo Jannone da Silva, doutorando, FOB-USP, Brasil <eduardoadvsilva@gmail.com>

Natália Barreto Frederigue Lopes, professora doutora, FOB-USP, Brasil <natalia@frederigue.com.br>

Adriane Lima Mortari Moret, professora associada, FOB-USP, Brasil < dri.m@fob.usp.br>

Regina Tangerino de Souza Jacob, professor doutora, FOB-USP, Brasil <reginatangerino@usp.br> 